Tropical Journal of Pharmaceutical Research July 2019; 18 (7): 1467-1473

ISSN: $1596-5996$ (print); 1596-9827 (electronic)

(C) Pharmacotherapy Group, Faculty of Pharmacy, University of Benin, Benin City, 300001 Nigeria.

\title{
Salvia miltiorrhiza injection ameliorates myocardial ischemia-reperfusion injury via downregulation of PECAM-1
}

\author{
Yang Ronghai ${ }^{1}$, Yao Weiping ${ }^{2}$, Liu YingFeng ${ }^{1 *}$, Wang Xuejun ${ }^{3}$, Liang \\ Jianguang ${ }^{4}$, Liu Jiachao ${ }^{4}$ \\ ${ }^{1}$ Department of Cardiovascular Medicine, Zhujiang Hospital of Southern Medical University, Guangzhou 510280, ${ }^{2}$ Department \\ of Respiration Medicine, ${ }^{3}$ Department of Neurology, ${ }^{4}$ Department of Cardiovascular Medicine, People's Hospital of Sanshui \\ District, Foshan City, Foshan 528100, Guangdong, China
}

*For correspondence: Email: liuyingfeng034@aliyun.com

Sent for review: 16 December 2017

Revised accepted: 15 June 2019

\begin{abstract}
Purpose: To investigate the effect of Salvia miltiorrhiza injection on myocardial ischemia-reperfusion injury and PECAM-1 related pathways.

Method: Male Wistar rats were used for establishment of myocardial ischemia-reperfusion model. The rats were randomly assigned to four groups: experimental group, low dose group (Salvia miltiorrhiza injection, $10 \mathrm{~mL} / \mathrm{kg} /$ day), moderate dose group (Salvia miltiorrhiza injection, $20 \mathrm{~mL} / \mathrm{kg} /$ day) and high dose group (Salvia miltiorrhiza injection, $40 \mathrm{~mL} / \mathrm{kg} /$ day). Myocardial ischemia-reperfusion model was established in the four groups. Evans-TTC staining was used to assess relative area of ischemiareperfusion injury. Blood samples were collected for assay of PECAM-1 expression using enzymelinked immunosorbent assay (ELISA). Fresh blood platelets were collected in all groups, and divided into two groups - control group (normal culture) and experimental group (Salvia miltiorrhiza injection). The expression of PECAM-1 in blood platelets was assayed using Western blot.

Result: Compared with the experimental group, Salvia miltiorrhiza injection ameliorated myocardial ischemia-reperfusion injury, and decreased the infarction area seen in Evans/TTC staining. PECAM-1 expression in blood was decreased by Salvia miltiorrhiza injection. Blood platelets dysfunction was induced after myocardial ischemia-reperfusion, and the level of PECAM-1 increased. However, Salvia miltiorrhiza injection treatment downregulated the expression of PECAM-1 after myocardial ischemiareperfusion.

Conclusion: Salvia miltiorrhiza injection maintains normal function of blood platelets and ameliorates myocardial ischemia-reperfusion injury by decreasing expression of PECAM-1.
\end{abstract}

Keywords: Salvia miltiorrhiza, PECAM-1, Blood platelets, Myocardium, Ischemia-reperfusion

This is an Open Access article that uses a fund-ing model which does not charge readers or their institutions for access and distributed under the terms of the Creative Commons Attribution License (http://creativecommons.org/licenses/by/4.0) and the Budapest Open Access Initiative (http://www.budapestopenaccessinitiative.org/read), which permit unrestricted use, distribution, and reproduction in any medium, provided the original work is properly credited.

Tropical Journal of Pharmaceutical Research is indexed by Science Citation Index (SciSearch), Scopus, International Pharmaceutical Abstract, Chemical Abstracts, Embase, Index Copernicus, EBSCO, African Index Medicus, JournalSeek, Journal Citation Reports/Science Edition, Directory of Open Access Journals (DOAJ), African Journal Online, Bioline International, Open-J-Gate and Pharmacy Abstracts

\section{INTRODUCTION}

Myocardial ischemia-reperfusion (I/R) injury refers to aggravated myocardial damage which occurs during recovery of blood flow; and it exacerbates development of heart failure [1]. Previous clinical trials have shown that, with development of medical technology, more and more patients with ischemic heart disease now receive more effective treatments such as dual 
anti-platelet therapy, PCl and CABG [2]. However, the efficacies of these therapies are always abrogated by subsequent myocardial ischemia reperfusion injury. A recent study reported that ischemia-reperfusion injury worsened post-PCl prognosis and increased incidence of re-hospitalization [3]. Moreover, several mechanisms are involved in the development of myocardial ischemia reperfusion injury. These include oxygen free radicals, calcium overload, inflammatory response and mitochondria dysfunction [4 - 6]. However, the therapies targeting these mechanisms are not very effective. Thus, there is need to develop novel strategies for better understanding of myocardial ischemia reperfusion and more efficient treatment [7]. A recent study showed that, although the detailed mechanism is still unclear, blood platelets play an important role in the pathogenesis of $\mathrm{I} / \mathrm{R}$ besides their traditional effects on myocardial ischemia, suggesting that they could be novel targets for treatment of myocardial I/R [8].

Salvia miltiorrhiza injection (also known as Danshen injection) is a compound medicine with effective components extracted from the traditional Chinese medicinal herb Salvia miltiorrhiza [9]. Salvia miltiorrhiza injection has been shown to exhibit promising effects on chronic heart failure, and animal experiment showed that Salvia miltiorrhiza injection protected the myocardium via improving blood supply [10, 11]. Moreover, recent clinical trials demonstrated that Salvia miltiorrhiza injection improved cardiac function and ameliorated myocardial injury in patients with acute myocardial infarction [12], suggesting that the drug could influence progress of myocardial ischemia-reperfusion injury. It has been reported that Salvia miltiorrhiza injection stabilized the function of blood platelets [13]. However, the mechanisms involved is not yet understood.

Platelet endothelial cell adhesion molecule (PECAM-1, also known as CD31) is highly expressed in blood platelets. Studies have revealed that PECAM-1 is involved in heart development, and in adults, PECAM-1 influences the prognosis of cardiovascular disease via inflammatory response and the regulation of endothelium integrity [14]. Increased expression of PECAM-1 has been associated with poor prognosis of myocardial infarction [15]. Thus, PECAM-1 could be a therapeutic target for ischemia-reperfusion injury.

Accordingly, Salvia miltiorrhiza injection may attenuate myocardial ischemia-reperfusion injury, and PECAM-1 may be the down-stream target of Salvia miltiorrhiza injection.

\section{EXPERIMENTAL}

\section{Experimental animals and grouping}

A total of 80 male Wistar rats aged 8-10 weeks were purchased from Experimental Animal Center of Zhejiang Chinese Medical University [license number: SCXK (Zhe) 2015-0004]. The rats were kept in separate cages with animal laboratory temperature range of $20-25{ }^{\circ} \mathrm{C}$, and air relative humidity of $50-60 \%$. The experimental animals were handled in line with animal ethical standards. The I/R model rats were randomly assigned to four groups: experimental group, low dose group (Salvia miltiorrhiza injection $(10 \mathrm{~mL} / \mathrm{kg} /$ day for one week before I/R), moderate dose group (Salvia miltiorrhiza injection at a dose of $20 \mathrm{~mL} / \mathrm{kg} /$ day for 1 week before I/R) and high dose group (Salvia miltiorrhiza injection at a dose of 40 $\mathrm{mL} / \mathrm{kg} /$ day for 1 week before $\mathrm{l} / \mathrm{R})$. All experiments on rats were performed according to international guidelines for animal studies [16].

\section{Reagents}

Salvia miltiorrhiza injection was bought from Shanghai Boulder Biotechnology Company and Nantong Jinhua Pharmaceutical Group Company (Item No. H32020869), and was pre-processed according to routine protocols of Traditional Chinese Medicine [17]. Primary antibody for PECAM-1 was purchased from Shanghai Yanjing Biotechnology Company. Rodent ventilator was product of Shanghai Kemin Biotechnology Company.

\section{Isolation of blood platelets and grouping}

Blood platelets were isolated from blood samples of male Wistar male in the four groups, and also from healthy rats. The blood samples were centrifuged for $10 \mathrm{~min}$ at $1200 \mathrm{rpm}$. The following solutions were added: TYRODE buffer (138 $\mathrm{mmol} / \mathrm{L}), \mathrm{NaCl}(3.3 \mathrm{mmol} / \mathrm{L}), \mathrm{NaH}_{2} \mathrm{PO}_{4}$ (2. 9 $\mathrm{mmol} / \mathrm{L}), \mathrm{KCl}(1 \mathrm{mmol} / \mathrm{L}), \mathrm{MgCl}_{2}(5.5 \mathrm{mmol} / \mathrm{L})$, glucose (20 mmol/L), HEPES, pH 7.2, ACD (2.5 $\%$ ), trisodium citrate, $2.0 \%$ glucose, $1.5 \%$ citric acid, and $2 \mathrm{mmol} / \mathrm{L}$ EDTA. The mixture was centrifuged for $10 \mathrm{~min}$ at $1200 \mathrm{rpm}$, and the supernatant was discarded. The blood platelets were centrifuged and rinsed twice. The blood platelets from the experimental group were divided into two groups: control platelet group treated with normal medium; and injection. Ethical approval was obtained from Zhujiang 
Hospital of Southern Medical University (approval no. URSB741).

\section{Animals and grouping}

The animal model of $\mathrm{I} / \mathrm{R}$ was established as reported earlier [18]: After anaesthetizing the rats with intraperitoneal injection of $75 \mathrm{mg} / \mathrm{kg}$ ketamine and $7.5 \mathrm{mg} / \mathrm{kg}$ diazepam, oral endotracheal intubation was provided for assisted respiration of the rats on the plate. Then, the chest was opened along the midline to expose the heart. The left anterior descending branch in each experimental group rat was ligated using 6-0 mersilk within $30 \mathrm{~min}$. The rats were randomly allocated to IL-37 treatment (experimental group), and saline control (control group), with 10 rats in each group. The chest of other 10 sham rats were only opened and uncovered, but no ligation was carried out. Then, $1 \mu \mathrm{g}$ of human recombinant IL-37 (dissolved in $200 \mu \mathrm{L} 0.9 \% \mathrm{NaCL}$ ) was injected into the experimental group via the tail veins. The rats in the control group were injected with $200 \mu \mathrm{L}$ of $0.9 \% \mathrm{NaCL}$ through the tail veins. Each group of rats was sacrificed after $24 \mathrm{~h}$.

\section{ELISA for PECAM-1 in blood}

The rats were anesthetized with intraperitoneal injection of trichloroaldehyde $(60 \mathrm{mg} / \mathrm{kg})$. Then, blood was phlebotomized and centrifuged for ELISA determination of PECAM-1. Rat serum $(100 \mu \mathrm{L})$ and $100 \mu \mathrm{L}$ PBS (pH 7.4) were put into ELISA plate and allowed to react at $4{ }^{\circ} \mathrm{C}$ overnight. The plate was rinsed with PBS and blocked with $2 \% \mathrm{H}_{2} \mathrm{O}_{2}$-enthol. Then, $1 \% \mathrm{BSA}$ was added to block non-specific antibody binding sites, followed by rinsing of the mixture with PBS. Thereafter, PECAM-1-specific antibody (1:1000 dilution) was added and allowed to incubate for 2 $h$ at $37^{\circ} \mathrm{C}$, followed by incubation with goat antirat secondary antibody (1:200 dilution) for $1.5 \mathrm{~h}$ at $37{ }^{\circ} \mathrm{C}$. The reaction was stopped with OPD developing solution in $0.2 \mathrm{mM} \mathrm{H}_{2} \mathrm{SO}_{4}$. The quantity of PECAM-1 was detected using a microplate reader (Biotek).

\section{Assessment of myocardial ischemia/ reperfusion infarction area}

Echocardiography was performed after myocardial ischemia reperfusion, and ejection fraction (EF) and short axial shortening rate (FS) were determined. The rats were sacrificed thereafter, so as to stop their heartbeat in ventricular diastolic stage. The chest region of each rat was opened up. The left anterior descending branch was ligated again, and $1 \mathrm{~mL} 1$ $\%$ EVANS was injected along the aorta into chambers of the heart. The non-ischemic areas were dyed blue, while the ischemic areas were undyed. The heart was cut into $1 \mathrm{~mm}$ sections which were placed in 1\% TTC solution and incubated at $37{ }^{\circ} \mathrm{C}$ for $20 \mathrm{~min}$. The myocardial ischemic area was dyed blue. However, the myocardial infarction area was white, while the area of relative ischemic was dyed red. The areas of myocardial ischemia and infarction were detected with Image Pro Plus software. The ratio of myocardial ischemic area was calculated by dividing the weight of myocardial ischemic area with the weight of the left ventricle. The ratio of myocardial infarction was obtained by dividing the weight of myocardial infarction area with the weight of myocardial ischemia.

\section{Western blot}

Western blot was performed to determine the expression of PECAM-1 in blood platelets. The cells were lysed with RIPA Lysis buffer. Protein was transferred to PVDF membrane after SDSpolyacrylamide gel electrophoresis. Non-specific binding sites in the PVDF membrane were blocked with defatted milk (5\%) prior to incubation with PECAM-1 antibody. The internal control was GAPDH. Specific protein bands were detected with chemiluminescence.

\section{Statistical analysis}

Data are presented as mean \pm standard deviation (SD). Statistical analysis was done with $t$-test, using SPSS 20.0 software. $P<0.05$ were assumed to indicate statistical significance.

\section{RESULTS}

\section{Salvia miltiorrhiza injection reduced myocardial ischemia/reperfusion infarction area}

Evans/TTC staining showed that, when compared with experimental group, myocardial injury was attenuated after myocardial $\mathrm{l} / \mathrm{R}$ in all Salvia miltiorrhiza injection groups $(p<0.05$; Figure 1A). Moreover, in the Salvia miltiorrhiza injection groups, the moderate group (20 $\mathrm{mL} / \mathrm{kg} /$ day) had the smallest area of infarction ( $p$ $<0.05$; Figure 1A). However, there were no significant difference between the low dose group and high dose group $(p>0.05)$. These data suggest that Salvia miltiorrhiza injection protects the heart against I/R injury. Statistical analysis demonstrated that moderate dose treatment of Salvia miltiorrhiza injection produced the lowest percentage infarction $(p>$ 0.05 ; Figure $1 \mathrm{~B}$ ), consistent with staining data. 

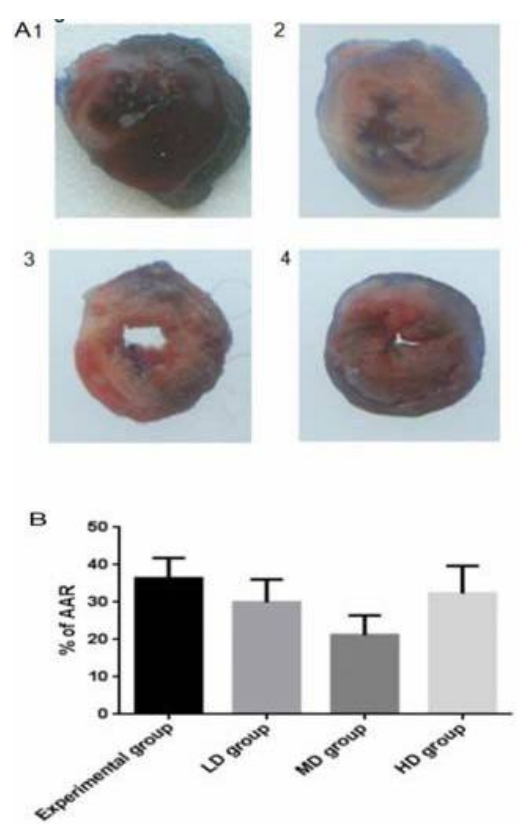

Figure 1: (A) Evans blue/TTC staining image for ischemic/reperfusion injury. 1: experimental group; 2 : low dose Salvia miltiorrhiza injection group; 3: moderate dose Salvia miltiorrhiza injection group; 4: high dose Salvia miltiorrhiza injection group. (B) Results of analysis of Evans blue/TTC staining. LD, MD and HD are low dose, medium dose and high dose Salvia miltiorrhiza injection groups, respectively

Salvia miltiorrhiza injection decreased the level of PECAM-1 in blood after myocardial ischemia/reperfusion

The expressions of PECAM-1 in all Salvia miltiorrhiza injection groups were significantly decreased $(p<0.05)$, when compared with the experimental group (Figure 2), suggesting that Salvia miltiorrhiza injection reduced myocardial ischemia/reperfusion injury via regulation of PECAM-1 expression. The high dose group had the lowest level of PECAM-1 $(p<0.05)$, suggesting that the effect of Salvia miltiorrhiza injection on PECAM-1 was dose-dependent.

\section{Myocardial ischemia/reperfusion enhanced the expression of PECAM-1}

Compared with blood platelets from healthy rats, PECAM-1 expression was increased in blood platelets from the experimental group (Figure 3). The data demonstrate that myocardial ischemia/reperfusion induced dysfunction of blood platelets, and upregulated the expression of PECAM-1 after myocardial ischemia/ reperfusion.
Salvia miltiorrhiza injection improved function of blood platelets after myocardial ischemia/reperfusion

As shown in Figure 3, blood platelets form Salvia miltiorrhiza injection groups had lower levels of PECAM-1 than blood platelets from experimental group. These data suggest that Salvia miltiorrhiza injection was associated with improvement of blood platelet function.

Salvia miltiorrhiza injection treatment ameliorated myocardial ischemia/reperfusioninduced dysfunction of blood platelets

Results from Western blot showed that Salvia miltiorrhiza injection decreased the expression of PECAM-1 (Figure 4), suggesting that it maintained normal functions of blood platelets via regulating PECAM-1.

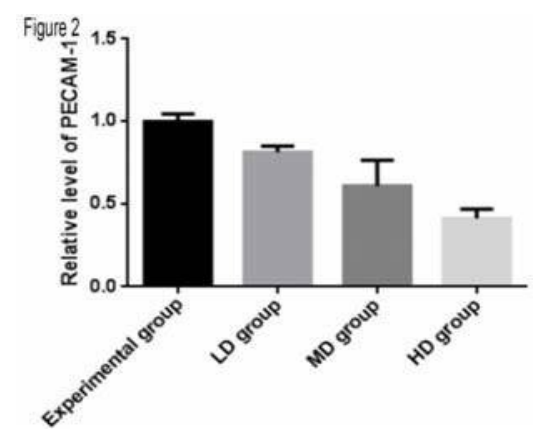

Figure 2: Blood levels of PECAM-1 in the various Salvia miltiorrhiza injection groups. LD, MD and HD are low dose, medium dose and high dose Salvia miltiorrhiza injection groups, respectively

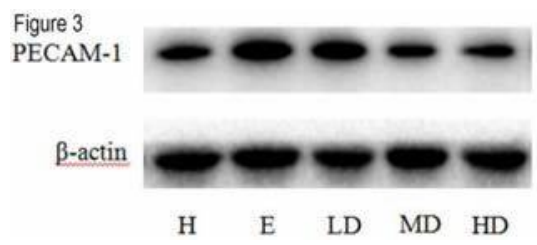

Figure 3: Western Blot for PECAM-1 in blood. $(\mathrm{H}=$ healthy rats; $\mathrm{E}+$ experimental group; $L D, M D$ and $H D$ are low dose, medium dose and high dose Salvia miltiorrhiza injection groups, respectively

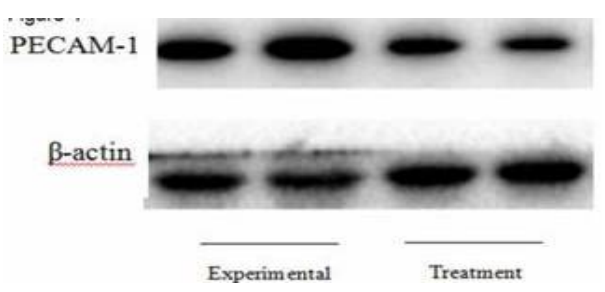

Figure 4: Western Blot for PECAM-1 on blood platelets. Experimental, blood platelets from experimental group; Treatment, Salvia miltiorrhiza injection-treated blood platelets from experimental group 


\section{DISCUSSION}

This study has demonstrated that Salvia miltiorrhiza injection is beneficial in the prevention of myocardial ischemia/reperfusion injury. Salvia miltiorrhiza injection decreased ischemia/reperfusion infarction area via maintaining normal function of blood platelets. Moreover, the results showed that Salvia miltiorrhiza injection regulated the expression levels of PECAM-1 in blood platelets. These results reveal the mechanism of action of Salvia miltiorrhiza, and suggest that PECAM-1 is a potential target for treatment of myocardial ischemia/reperfusion injury.

Salvia miltiorrhiza injection is a medicine widely used in clinics in China [19]. Previous clinical trials demonstrated that Salvia miltiorrhiza injection effectively improved prognosis of multiple cardiovascular diseases. However, the effect was mainly on chronic heart failure [20]. Although animal experiments have indicated that Salvia miltiorrhiza injection protected ischemic myocardialum [21], there is no concrete evidence for its effect on myocardial ischemia/reperfusion injury. The present study showed that Salvia miltiorrhiza injection attenuated myocardial ischemia/reperfusion injury, as was revealed via reduced infarction area. In addition, the results from staining experiments showed that moderate dose of Salvia miltiorrhiza injection produced optimal efficacy in the reduction of infarction area. Moreover, the inhibitory effect of Salvia miltiorrhiza injection on PECAM-1 production was dose-dependent. These evidence indicate that the therapeutic dose of Salvia miltiorrhiza injection should be adjusted to achieve optimal efficacy in the treatment of myocardial ischemia/reperfusion. The efficacy of Salvia miltiorrhiza injection can be partly explained through the blood platelet- and PECAM-1-related pathways. However, other mechanisms may be also be involved in this process, consistent with previous reports $[22,23]$.

Blood platelet dysfunction has been associated with myocardial ischemia/reperfusion [24]. Results from animal experiments have shown that the expressions of multiple markers were enhanced in blood platelets after ROS stimulation, and that PECAM-1 (one of these markers), exacerbated myocardial ischemia injury. Studies have also revealed that PECAM-1 influences the pathogenesis of myocardial ischemia/reperfusion [25]. In the present study, ELISA results showed that the expression of PECAM-1 in blood was increased after myocardial/reperfusion, while treatment with Salvia miltiorrhiza injection decreased PECAM-1 levels. Furthermore, blood platelet experiment showed that Salvia miltiorrhiza injection reduced ischemia/reperfusion-induced PECAM-1increase in blood platelets. Thus, Salvia miltiorrhiza injection maintains normal function of blood platelets via regulation of levels of PECAM-1, implying that Salvia miltiorrhiza injection may be a potential therapy for ischemia/reperfusion injury. Studies on Chinese traditional medicine have indicated that tanshinone is the most effective component in Salvia miltiorrhiza [23]. In addition, tanshinone has been shown to be efficacious not only on blood vessel endothelium, but also on blood platelets [24]. Accordingly, it may be hypothesized that tanshinone in Salvia miltiorrhiza injection is responsible for the observed regulation of PECAM-1 which resulted in maintenance of normal function of blood platelets in myocardial ischemic/reperfusion. However, further studies are needed to determine the exact role of tanshinone in this process.

\section{CONCLUSION}

Salvia miltiorrhiza injection is an effective therapy for the management of myocardial ischemia/reperfusion injury. It maintains the normal function of blood platelets by decreasing expression of PECAM-1, thereby ameliorating myocardial ischemia-reperfusion injury.

\section{DECLARATIONS}

\section{Acknowledgement}

This work was supported by Key Medical Project of the Foshan Municipal Science and Technology Bureau (no. 2018AB000945).

\section{Conflict of interest}

No conflict of interest is associated with this work.

\section{Contribution of authors}

We declare that this work was done by the authors named in this article and all liabilities pertaining to claims relating to the content of this article will be borne by the authors. Yang Ronghai and Yao Weiping carried out the experiments and did data analysis. Liu YingFeng designed the study, and took part in the data analysis. Wang Xuejun and Liang Jianguang took part in performing the experiments. Liu Jiachao was in the data analysis.

\section{Open Access}


This is an Open Access article that uses a funding model which does not charge readers or their institutions for access and distributed under the terms of the Creative Commons Attribution License (http://creativecommons.org/licenses/by/ 4.0) and the Budapest Open Access Initiative (http://www.budapestopenaccessinitiative.org/rea d), which permit unrestricted use, distribution, and reproduction in any medium, provided the original work is properly credited.

\section{REFERENCES}

1. Heusch G, Rassaf T. Time to Give Up on Cardioprotection? A Critical Appraisal of Clinical Studies on Ischemic Pre-, Post-, and Remote Conditioning. Circ Res 2016; 119(5): 676-695.

2. Rossello $X$, Yellon DM. A critical review on the translational journey of cardioprotective therapies! Int $J$ Cardiol 2016; 220: 176-184.

3. Andrew J, Macdonald P. Latest developments in heart transplantation: a review. Clin Ther 2015; 37(10): 22342241.

4. Makhdoumi P, Roohbakhsh A, Karimi G. MicroRNAs regulate mitochondrial apoptotic pathway in myocardial ischemia-reperfusion-injury. Biomed Pharmacother 2016; 84: 1635-1644.

5. Yang CJ, Yang J, Fan ZX, Yang J. Activating transcription factor 3--an endogenous inhibitor of myocardial ischemia-reperfusion injury (Review). Mol Med Rep 2016; 13(1): 9-12.

6. Le Page S, Bejan-Angoulvant T, Angoulvant D, Prunier $F$. Remote ischemic conditioning and cardioprotection: a systematic review and meta-analysis of randomized clinical trials. Basic Res Cardiol 2015; 110(2): 11.

7. Thind GS, Agrawal PR, Hirsh B, Saravolatz L, ChenScarabelli C, Narula J, Scarabelli TM. Mechanisms of myocardial ischemia-reperfusion injury and the cytoprotective role of minocycline: scope and limitations. Future Cardiol 2015; 11(1): 61-76.

8. Meyer AS, Ostrowski SR, Kjaergaard J, Johansson PI, Hassager C. Endothelial Dysfunction in Resuscitated Cardiac Arrest (ENDO-RCA): safety and efficacy of lowdose prostacyclin administration and blood pressure target in addition to standard therapy, as compared to standard therapy alone, in post-cardiac arrest syndrome patients: study protocol for a randomized controlled trial. Trials 2016; Aug 2;17: 378.

9. Shen Y, Hou J, Deng W, Feng Z, Yang M, Cheng J, Wu W, Guo DA. Comparative Analysis of Ultrafine Granular Powder and Decoction Pieces of Salvia miltiorrhiza by UPLC-UV-MSn Combined with Statistical Analysis. Planta Med 2016; Dec 19.

10. You JS, Pan TL, Lee YS. Protective effects of Danshen (Salvia miltiorrhiza) onadriamycin-induced cardiac and hepatic toxicity in rats. Phytother Res 2007; 21(12): 1146-1152.
11. Li DP, Chen Q, Yi L. Effects of yiqi huoxue method on cardiac function in patients with congestive heart failure. Zhongguo Zhong Xi Yi Jie He Za Zhi 2006; 26(6): 552554.

12. Ai F, Chen M, Li W, Yang Y, Xu G, Gui F, Liu Z, Bai X, Chen Z. Danshen improves damaged cardiac angiogenesis and cardiac function induced by myocardial infarction by modulating HIF1a/VEGFA signaling pathway. Int J Clin Exp Med 2015; 15;8(10): 18311-18318.

13. Liu L, Li J, Zhang Y, Zhang S, Ye J, Wen Z, Ding J, Kunapuli SP, Luo $X$, Ding $Z$. Salvianolic acid $B$ inhibits platelets as a P2Y12 antagonist and PDE inhibitor: evidence from clinic to laboratory. Thromb Res 2014; 134(4): 866-876.

14. Pamuk ON, Tozkir H, Uyanik MS, Gurkan H, Saritas $F$, Duymaz J, Donmez S, Yazar M, Pamuk GE. PECAM-1 gene polymorphisms and soluble PECAM-1 level in rheumatoid arthritis and systemic lupus erythematosus patients: any link with clinical atherosclerotic events? Clin Rheumatol 2014; 33(12): 1737-1743.

15. Dorland YL, Huveneers S. Cell-cell junctional mechanotransduction in endothelial remodeling. Cell Mol Life Sci 2017; 74(2): 279-292.

16. Rehbinder $C$, Baneux $P$, Forbes $D$, van Herck $H$, Nicklas W, Rugaya Z, Winkler G. FELASA recommendations for the health monitoring of mouse, rat, hamster, gerbil, guinea pig and rabbit experimental units. Report of the Federation of European Laboratory Animal Science Associations (FELASA) Working Group on Animal Health accepted by the FELASA Board of Management, November 1995.Lab. Anim. 1996 Jul; 30(3).

17. Li X, Wang $X Y$, Wu WQ, Zhao JF, Li JM. Study on prescription screening and preparation process of compound danshen pulse capsule. Zhongguo Zhong Yao Za Zhi 2013; 38(24): 4309-4313.

18. Bian B, Yu XF, Wang GQ, Teng TM. Role of miRNA-1 in regulating connexin 43 in ischemia-reperfusion heart injury: a rat model. Cardiovasc Pathol 2017; 27: 37-42.

19. Liu Y, Huang Y, Zhao C, Qin X, Zhu Q, Chen S, Qu J. Salvia miltiorrhiza injection on pulmonary heart disease: a systematic review and meta-analysis. Am J Chin Med 2014; 42(6): 1315-1331.

20. Chen J, Deng J, Zhang Y, Yang J, He Y, Fu W, Xing P, Wan Ht. Lipid-lowering effects of Danhong injection on hyperlipidemia rats. J Ethnopharmacol 2014; 154(2): 437-442.

21. Han B, Zhang $X$, Zhang Q, Zhao G, Wei J, Ma S, Zhu W, Wei M. Protective effects of salvianolate on microvascular flow in a porcine model of myocardial ischaemia and reperfusion. Arch Cardiovasc Dis 2011; 104(5): 313-324.

22. Huang W, Yang Y, Zeng Z, Su M, Gao Q, Zhu B. Effect of Salvia miltiorrhiza and ligustrazine injection on myocardial ischemia/reperfusion and hypoxial reoxygenation injury. Mol Med Rep 2016; 14(5): 45374544. 
23. Duan ZZ, Li YH, Li YY, Fan GW, Chang YX, Yu B, Gao $X M$. Danhong injection protects cardiomyocytes against hypoxia/reoxygenation- and $\mathrm{H}_{2} \mathrm{O}_{2}$-induced injury by inhibiting mitochondrial permeability transition pore opening. J Ethnopharmacol 2015; 175: 617-625.

24. Ergatoudes C, Thunström E, Rosengren A, Björck $L$, Bengtsson Boström K, Falk K, FuM. Long-term secondary prevention of acute myocardial infarction
(SEPAT) - guidelines adherence and outcome. BMC Cardiovasc Disord 2016; 16(1): 226.

25. Yoshizumi T, Zhu Y, Jiang H, D'Amore A, Sakaguchi $H$, Tchao J, Tobita $K$, Wagner WR. Timing effect of intramyocardial hydrogel injection for positively impacting left ventricular remodeling after myocardial infarction. Biomaterials 2016; 83: 182-193. 